\title{
Screening of Lactobacillus spp, for mediating the biosynthesis of silver nanoparticles from silver nitrate.
}

\author{
Ranganath $\mathrm{E}^{1}$, Vandana Rathod ${ }^{2}$ and Afreen Banu ${ }^{3}$ \\ ${ }_{1,2,3}$ (Department of Microbiology/ Gulbarga University, India)
}

\begin{abstract}
In modern nano science and technology the interaction between inorganic nanoparticles and biological structures are the most exciting areas of research. The desire to synthesize nanoparticles, using efficient and green chemistry approaches has led to the use of microorganisms. Hence the present study reports on the screening of potent Lactobacillus spp for ecofriendly and economical synthesis of silver nanoparticles. Total of nine Lactobacillus strains were isolated from milk and milk products inoculated to sterilized milk and latter (after 24hr) the whey was collected by coarse filtration (Whatman no.40). Silver nitrate $(1 \mathrm{mg})$ was added to $5 \mathrm{ml}$ of pale yellow filtrate then incubated at $37^{\circ} \mathrm{C}$. Absorption maximum was measured using spectrophotometer. The silver nanoparticles of size ranging from 2 to $20 \mathrm{~nm}$ were synthesized by Lactobacillus sp VRS-2 which was confirmed by TEM and EDS analysis. Thus, rapid and ecofriendly biosynthesis of silver nanoparticles using whey appears to be economical approach and any research in this direction is encouraging.
\end{abstract}

Key words- Antimicrobial resistance, Lactobacillus spp, Nanobiotechnology. Silver nanoparticles,

\section{Introduction}

There is a growing interest in nanoparticles as they provide superior material properties with functional versatility. Especially Inorganic nano materials have been widely used for cellular delivery due to their versatile features like wide availability, rich functionality, good biocompatibility, capability of targeted drug delivery and controlled release of drugs [1]. Silver nanoparticles are among the most commercialized inorganic nanoparticles due to their antimicrobial potential. They have also been used for a number of applications such as non linear optics, spectrally selective coating for solar energy absorption, biolabelling and antibacterial activities.

Since biocide-resistant strains emerged, the interest to use silver as an antimicrobial agent is rising again [2]. However, antimicrobial agents based on ionic silver (e.g., silver nitrate) have one major drawback, that they are easily inactivated by complexation and precipitation and thus have a limited usefulness [3].Zerovalent silver nanoparticles are considered as a valuable alternative for ionic silver. Due to their large specific surface-to-volume ratio, nanoparticles have different properties than bulk material [4]. It has been shown that silver nanoparticles are antimicrobial towards a broad spectrum of Gram-negative and Gram-positive bacteria [5, 6]. Furthermore, silver nanoparticles show antifungal [7] and antiviral activity [8].

Production of silver nanoparticles can be achieved through different methods. Chemical approaches are the most popular methods for the production. However, some chemical methods cannot avoid the use of toxic chemicals in the synthesis protocol. Since noble metal nanoparticles such as silver, gold nanoparticles are widely applied to human contacting areas. There is a growing need to develop environmentally friendly processes of nanoparticles synthesis that do not use toxic chemicals [9]. The biomedical applications of silver nanoparticles can be effective by the use of biologically synthesized nanoparticles which minimize the factors such as toxicity and cost and are found to be exceptionally stable.

The formation of extracellular and intracellular silver nanoparticles by bacteria (Pseudomonas stulzeri, Escherichia coli, Vibrio cholerae, Pseudomonas aeruginosa, Salmonella typhus, and Staphylococcus currens) has been investigated [10]. There is a need to develop an ecofriendly approach for nanomaterial synthesis that should not use toxic chemicals in the synthesis protocol and use of microbes which are Generally Regarded As Safe (GRAS). Hence in the present study, the isolation and screening of Lactobacillus spp (Gram-positive commensal inhabitants of the gastrointestinal tract that also play important roles in the production and preservation of food) was carried to explore their capabilities of biosynthesizing silver nanoparticles which have exceptional antimicrobial activity against broad spectrum Gram-negative and Gram-positive bacteria.

\section{Materials and methods}

\subsection{Isolation of Lactobacillus spp}

The sample like curd, milk, cheese and khoa, were collected from various local vendors and retail shops. Lactobacillus strains used in the study were isolated by serial dilution technique using Modified Lactobacillus Agar (Hi-media, India). The bacterium was identified based on cultural and biochemical characteristics. Determination of the isolates was performed according to their morphological, cultural, biochemical and physiological characteristics by the procedures described in Bergey's Manual of Systematic Bacteriology [11]. Initially all of the isolates were examined for Gram staining and catalase production. Then cell morphology and colony characteristics on MRS agar (deMan Rogosa and Sharpe agar) were tested and 
the isolates were separated into different phenotypic groups. Only Gram-positive, catalase-negative and rod shaped isolates were selected for further studies. The isolates were maintained in slants of tomato juice agar.

\subsection{Screening of Lactobacillus spp for silver nanoparticle synthesis}

In a typical procedure of nanoparticles synthesis, every isolate were individually inoculated into sterilize $250 \mathrm{ml}$ of home delivered milk in $500 \mathrm{ml}$ Erlenmeyer flask and incubated for curdling at $37^{\circ} \mathrm{C}$ for 24 hours. The whey was collected by coarse filtration (Whatman 40). The filtrate was pale yellow in appearance, and the $\mathrm{pH}$ was typically 4.4. The presence of lactic acid bacteria in the supernatant was confirmed by observation taken from optical microscope. To $5 \mathrm{~mL}$ of each sample solution taken in a test tube, $1 \mathrm{mg}$ of $\mathrm{AgNo}_{3}$ was added and kept in the laboratory under ambient conditions [12]. The solution became brown in about $12 \mathrm{~h}$. A brown mass gets deposited at the bottom of the test tube after $24 \mathrm{~h}$. Control was run along with experimental flask.

\subsection{Characterization of silver nanoparticles}

The reaction mixture was analyzed periodically using UV-Vis spectrophotometer (Thermo Spectronic Genesys 10 UV Spectrophotometer). The absorbance was measured in the range 300-600 nm, which includes the Plasmon absorbance peak of the silver nanoparticles centered at $420 \mathrm{~nm}$. Further the nanoparticles from the potent isolate were characterized by Transmission Electron Microscopic (TEM) and Energy Disperse Spectroscope (EDS) analysis.

A drop of sample was placed on a piece of parafilm, carbon coated copper grid was placed and allowed for 5-10 minutes, and drained the excess with help of filter paper. Further again the preparation was washed with distilled water and stained with $2 \%$ uranyl acetate. The dried preparation was observed under transmission electron microscope at various magnifications (Model: Hitachi, H-7500) as per the standard protocol [13]. The presence of elemental silver was confirmed through Energy Disperse Spectroscopy.

\section{Results and Discussions}

\subsection{Production of silver nanoparticles}

In the present study among 9 isolates of Lactobacillus spp, one isolate designated as VRS-2 was primarily confirmed as positive by change of the reaction mixture from pale yellow to brown color (Fig 1) indicating the production of silver nanoparticles $\left(\mathrm{Ag}^{+}\right.$to $\left.\mathrm{Ag}^{0}\right)$. It is reported that reduction of $\mathrm{Ag}^{+}$to $\mathrm{Ag}^{0}$ occurs through nitrate reductase enzyme [14]. These enzymes released in the solution can reduce the silver nitrate to silver nanoparticles through capping agents such as protein s. Brown colored precipitate was further employed for characterization of silver nanoparticles.

\subsection{Characterization of silver nanoparticles}

The reaction mixture was analyzed using UV-Vis spectrophotometer. The absorbance was measured in the range 300-650nm, which includes the Plasmon absorbance peak of the silver nano particles centered at $430 \mathrm{~nm}$ (Fig-2). The silver nanoparticles formed were highly stable even after few weeks after the reaction [9]. Formation of colloidal silver particles can be easily followed by changes of UV-Vis absorption [15] optical absorption spectroscopy has proved to be a very useful technique for the analysis of nanoparticles. The characteristic brown color due to the excitation of Plasmon vibrations in the nanoparticles provides a convenient signature of their formation [16]. Appearance of colour arises from the property of the colored material to absorb selectively within the visible region of the electromagnetic spectrum [17]. The surface Plasmon band remains in the range of 420-440 $\mathrm{nm}$ throughout the reaction period suggesting that the particles are dispersed in the aqueous solution with no evidence for aggregation after the complete of reaction.

TEM picture of the silver nanoparticles formed by Lactobacillus sps VRS-2 after $48 \mathrm{~h}$ is shown in Fig 3. The TEM Technique used to visualize size and shapes of biosynthesized silver nanoparticles have predominantly shown spherical shape structures with size ranging between $2 \mathrm{~nm}-20 \mathrm{~nm}$. This picture shows individual as well as number of aggregates. Nanoparticles formed were highly stable due to capping agents such as proteins, and were not in direct contact even within the aggregates indicating stabilization of the nanoparticles by a capping agent [18]. Reduction followed by bioadsorption of silver nanoparticles is evident after the analysis of TEM micrograph. The reduction of $\mathrm{Ag}^{+}$ions to elemental silver by Lactobacillus sps VRS-2 was further characterized by EDS analysis which gives the additional evidence for silver nanoparticle synthesized [19]. The optical absorption peak is observed approximately at $3 \mathrm{keV}$, which is typical for the absorption of metallic silver nanocrystals due to surface plasmon resonance, which confirms the presence of nanocrystalline elemental silver (Fig-4). Spectrum shows strong silver signal along with adjacent phosphorus peak. Nair and Pradeep [12] first reported that common Lactobacillus strains found in buttermilk assisted the growth of microscopic gold, silver, and gold-silver alloy crystals of well-defined morphology. Biosorption and bioreduction of $\mathrm{Ag}^{\circ}$ on cell surface was also reported in Lactobacillus sps. A09 at $30^{\circ} \mathrm{C}, \mathrm{pH} 4.5$ in $24 \mathrm{~h}$ [20].

\section{Conclusions}

Nanotechnology offers important new tools expected to have a great impact on many areas in medical technology. It is expected to accelerate scientific as well as economic activities in medical research and development. Nanotechnology has the potential to make significant contributions to disease detection, diagnosis, therapy, and prevention. In the present study we 
have reported a simple biological extracellular, easy, low cost, non toxic economical and ecofriendly approach for synthesizing silver nanoparticles by using Lactobacillus sps VRS-2, which provides extraordinary opportunities to improve materials and medical devices. The silver nanoparticles formed were characterized by UV-Vis spectra, TEM and EDS studies. The size of the particles was of $2-20 \mathrm{~nm}$. Our further studies will be focused on various medical applications of silver nano particles.

\section{Acknowledgements}

The authors are thankful to Gulbarga University for providing the facilities to carry out the present investigation in the Department of Microbiology, Gulbarga University, Gulbarga, Karnataka- India.

\section{Figures}

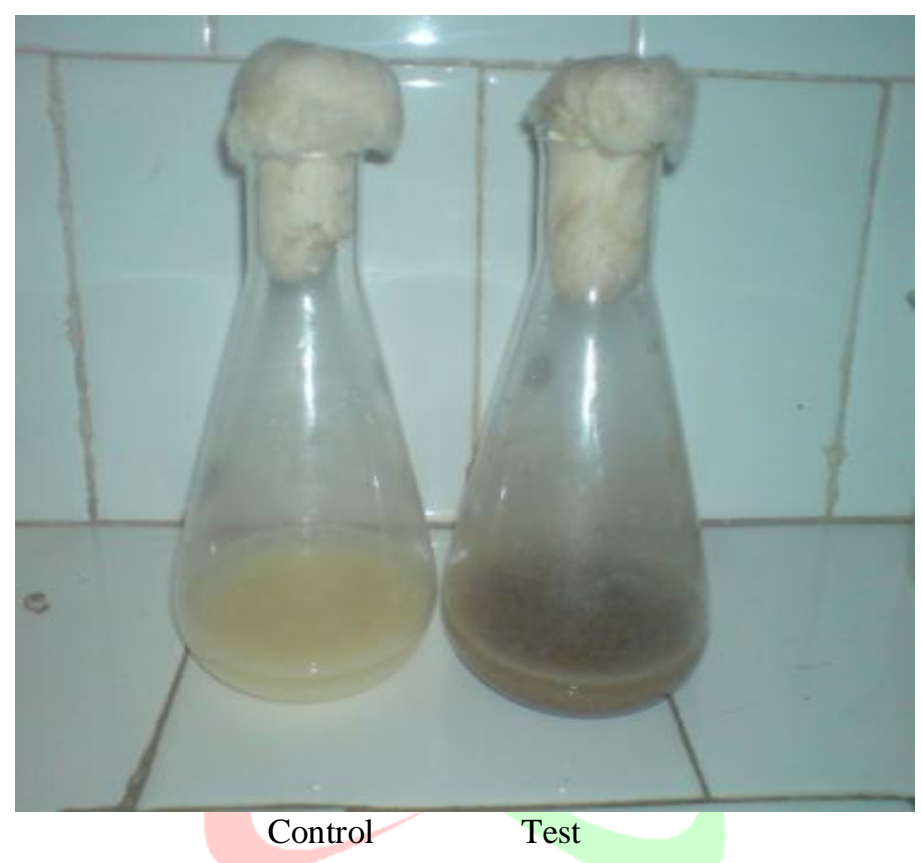

Figure 1: Lactobacillus sps VRS-2 in whey (a) without AgNO $\mathrm{H}_{3}$ taken as control (b) with $\mathrm{AgNO}(1 \mathrm{mg} / 5 \mathrm{ml})$

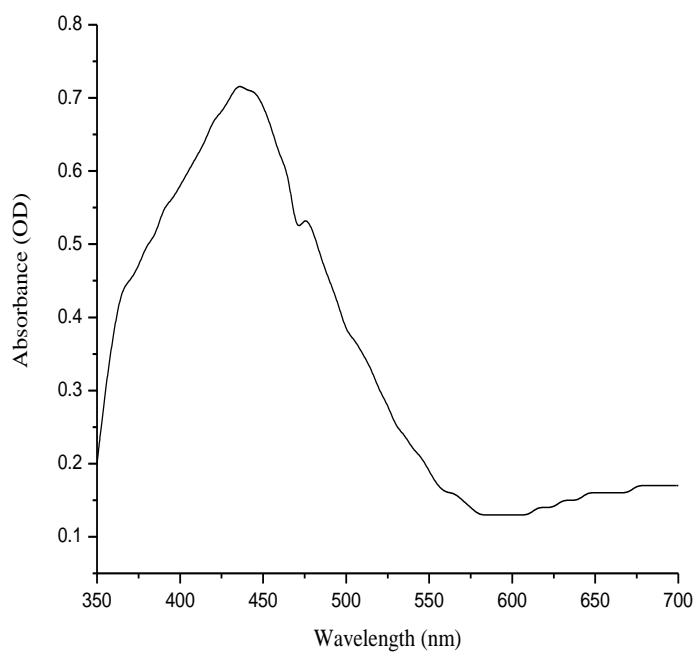

Figure 2: Absorbance spectrum of silver nanoparticles synthesized by Lactobacillus sps VRS-2 


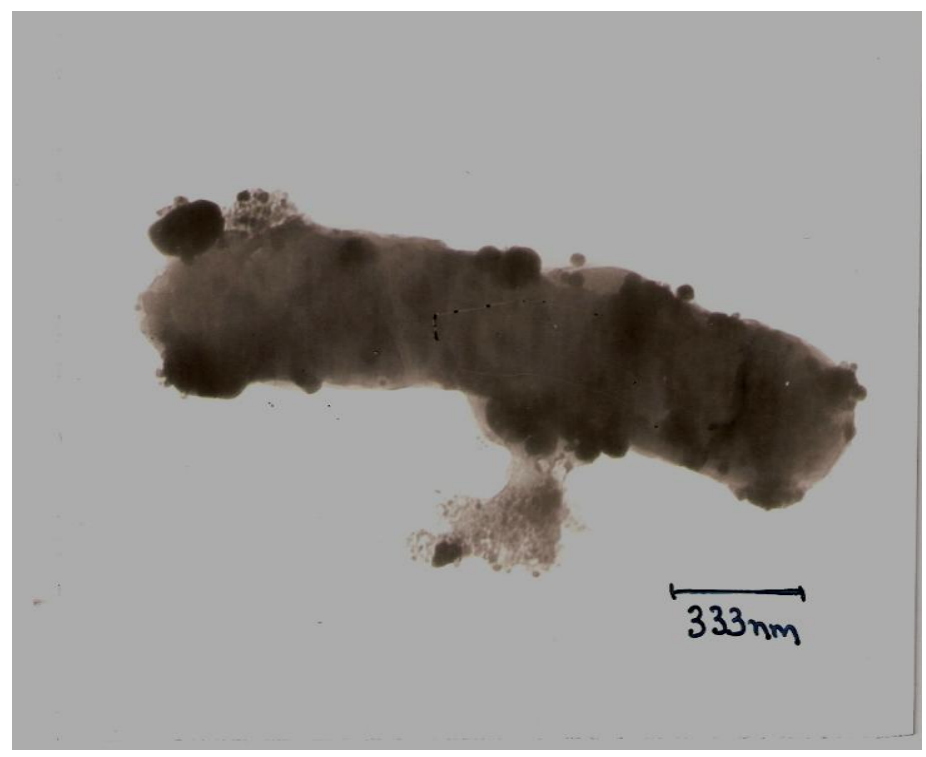

Figure 3: Transmission electron micrograph of silver nanoparticles embedded on Lactobacillus sps VRS-2

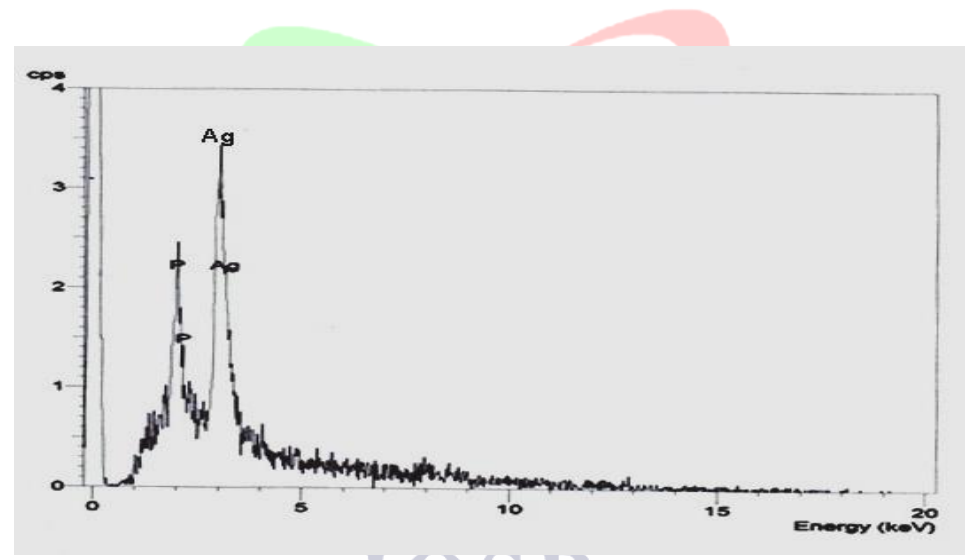

Figure 4: EDS of silver nanoparticles synthesized by Lactobacillus sps VRS-2

\section{References}

1. Z.P. Xu, Q.H. Zeng, G.Q. Lu and A.B. Yu, Inorganic nanoparticles as carriers for efficient cellular delivery. Chemical Engineering Science 61(3), 2006, 1027- 1040.

2. H.J. Klasen, Historical review of the use of silver in the treatment of burns. I. Early uses. Burns 26(2), 2000,117-130

3. B.S. Atiyeh, M. Costagliola, S.N. Hayek, S.A. Dibo, Effect of silver on burn wound infection control and healing: review of the literature. Burns 33(2), 2007, 139-148

4. R.M. Tilaki, A.I. Zad, S.M. Mahdavi, Stability, size and optical properties of silver nanoparticles prepared by laser ablation in different carrier media, Applied Physics A: Materials Science \& Processing, 84(1-2), 2006, 215- 219

5. A. Panacek, L. Kvitek, R. Prucek, M. Kolar, R. Vecerova, N. Pizurov a, V.K.Sharma, T. Nevecna, R. Zboril, Silver colloid nanoparticles: synthesis, characterization, and their antibacterial activity, Journal of Physical Chemistry B, 110(33), 2006, 16248-16253

6. J.S. Kim, E. Kuk, K.N. Yu, J.H. Kim, S.J. Park, H.J. Lee, S.H. Kim, Y.K. Park,Y.H. Park, C.Y. Hwang, Y.K. Kim, Y.S. Lee, D.H. Jeong and M.H. Cho, Antimicrobial effects of silver nanoparticles. Nanomedicine 3(1), 2007, 95-101

7. K.J. Kim, W.S Sung, S.K. Moon, J.S. Choi, J.G. Kim and D.G. Lee, Antifungal effect of silver nanoparticles on dermatophytes. Journal of Microbiology and Biotechnology, 18(8), 2008, 1482-1484

8. J.L. Elechiguerra, J.L. Burt, J.R. Morones, A. Camacho-Bragado, X. Gao, H.H. Lara and M.J. Yacaman, Interaction of silver nanoparticles with HIV-1. Journal of Nanobiotechnol 3:6, 2005. 
9. N. Duran, P.D. Marcato, O.L. Alves, G.I. Souza and E. Esposito, Mechanistic aspects of biosynthesis of silver nanoparticles by several Fusarium oxysporum strains. Journal of Nanobiotechnol 3:8, 2005.

10. F.M. Lengke, E.M. Fleet and G. Southam, Biosynthesis of silver nanoparticles by filamentous cyanobacteria a from a silver(I) nitrate complex, Langmuir, 23(5), 2007, 2694- 2699.

11. O. Kandler, and N. Weiss, Regular Nonsporing Gram-Positive Rods, in N.R. Krieg and J.G. Halt (Ed.), Bergey's Manual of systematic Bacteriology, (Baltimore: Williams and Wilkins, 1986) 1209-1234.

12. B. Nair and T. Pradeep, Coalescence of nanoclusters and formation of submicron crystallites assisted by Lactobacillus strains. Crystal Growth Design, 2(4), 2002, 293-298

13. J. John, Bozzola, and D. Russell Lonnie, Electron Microscopy Principles and Techniques for Biologists (Sudbury, Massachusetts, Jones and Bartlett, 1998).

14. M. Sastry, A. Ahmad, M.I. Khan and R. Kumar, Biosynthesis of metal nanoparticles using fungi and actinomycete. Current Sciences, 85(2), 2003, 162-170

15. Z. Sadowski, H.I. Maliszewska, B. Grochowalska, I. Polowczyk, T. Kozlecki, Synthesis of silver nanoparticles using microorganisms, Materials Science-Poland, 26(2), 2008, 419-424

16. F. G. Rutberg, M. V. Dubina, V. A. Kolikov, F. V. Moiseenko, E. V. Ignat'eva, N. M. Volkov, V. N. Snetov, and A. Y. Stogov, Effect of silver oxide nanoparticles on tumor growth in vivo, Doklady Biochemistry and Biophysics 421(1), 2008, 191-193.

17. B. Afreen, R. Vandana and E. Ranganath, Silver nanoparticle production by Rhizopus stolonifer and its antibacterial activity against extended spectrum b-lactamase producing (ESBL) strains of Enterobacteriaceae, Materials Research Bulletin 46(9), 2011, 1417-1423

18. A. Ahmad, P. Mukherjee, S. Senapati, D. Mandal, M.I. Khan, R. Kumar and M. Sastry, Extracellular biosynthesis of silver nanoparticles using the fungus Fusarium oxysporum, Colloids Surfaces, B: Biointerfaces, 28(4), 2003, 313-318.

19. M. F. Amanulla, K. Balaji, M. Girilal, Y. Ruchi, P.T. Kalaichelvan, R. Venketesan, Biogenic synthesis of silver nanoparticles and their synergistic effect with antibiotics: a study against gram-positive and gramnegative bacteria, Journal of Nanomedicine, 6(1), 2010, 103-109.

20. Z.Y. Lin, C.H. Zhou, J.M. Wu, J.Z. Zhou, L. Wang, Afurther insight into the mechanism of Ag+ biosorption by Lactobacillus sp strain A09. Spectrochimica Acta Part A: Molecular and Biomolecular Spectroscopy 61(6), 2005, 1195-1200. 